\title{
Predicting RNA Secondary Structures with Arbitrary Pseudoknots by Maximizing the Number of Stacking Pairs
}

\author{
Samuel Ieong* Ming-Yang Kao ${ }^{\dagger} \quad$ Tak-Wah Lam ${ }^{\ddagger}$ \\ Wing-Kin Sung ${ }^{\S} \quad$ Siu-Ming $\mathrm{Yiu}^{\ddagger}$
}

\begin{abstract}
The paper investigates the computational problem of predicting RNA secondary structures. The general belief is that allowing pseudoknots makes the problem hard. Existing polynomial-time algorithms are heuristic algorithms with no performance guarantee and can only handle limited types of pseudoknots. In this paper we initiate the study of predicting RNA secondary structures with a maximum number of stacking pairs while allowing arbitrary pseudoknots. We obtain two approximation algorithms with worst-case approximation ratios of $1 / 2$ and $1 / 3$ for planar and general secondary structures, respectively. For an RNA sequence of $n$ bases, the approximation algorithm for planar secondary structures runs in $O\left(n^{3}\right)$ time while that for the general case runs in linear time. Furthermore, we prove that allowing pseudoknots makes it NP-hard to maximize the number of stacking pairs in a planar secondary structure. This result is in contrast with the recent NP-hard results on psuedoknots which are based on optimizing some general and complicated energy functions.
\end{abstract}

\section{Introduction}

Ribonucleic acids (RNAs) are molecules that are responsible for regulating many genetic and metabolic activities in cells. An RNA is single-stranded and can be considered as a sequence of nucleotides (also known as bases). There are four basic nucleotides, namely, Adenine (A), Cytosine (C), Guanine (G), and Uracil (U). An RNA folds into a 3-dimensional structure by forming pairs of bases. Paired bases tend to stabilize the RNA (i.e., have negative free energy). Yet base pairing does not occur arbitrarily. In particular, A-U and C-G form stable pairs and are known as the Watson-Crick base pairs. Other base pairings are less stable and often ignored. An example of a folded RNA is shown in Figure 11. Note that this figure is just schematic; in practice, RNAs are 3-dimensional molecules.

\footnotetext{
*Department of Computer Science, Yale University, New Haven, CT 06520.

${ }^{\dagger}$ Department of Computer Science, Northwestern University, Evanston, IL 60201 (kao@cs.northwestern.edu). This research was supported in part by NSF Grant EIA-0112934.

${ }^{\ddagger}$ Department of Computer Science, The University of Hong Kong, Hong Kong (\{twlam, smyiu\}@cs.hku.hk). This research was supported in part by Hong Kong RGC grant HKU-7027/98E.

${ }^{\S}$ Department of Computer Science, National University of Singapore, 3 Science Drive 2, Singapore 117543 (ksung@comp.nus.edu.sg).
} 


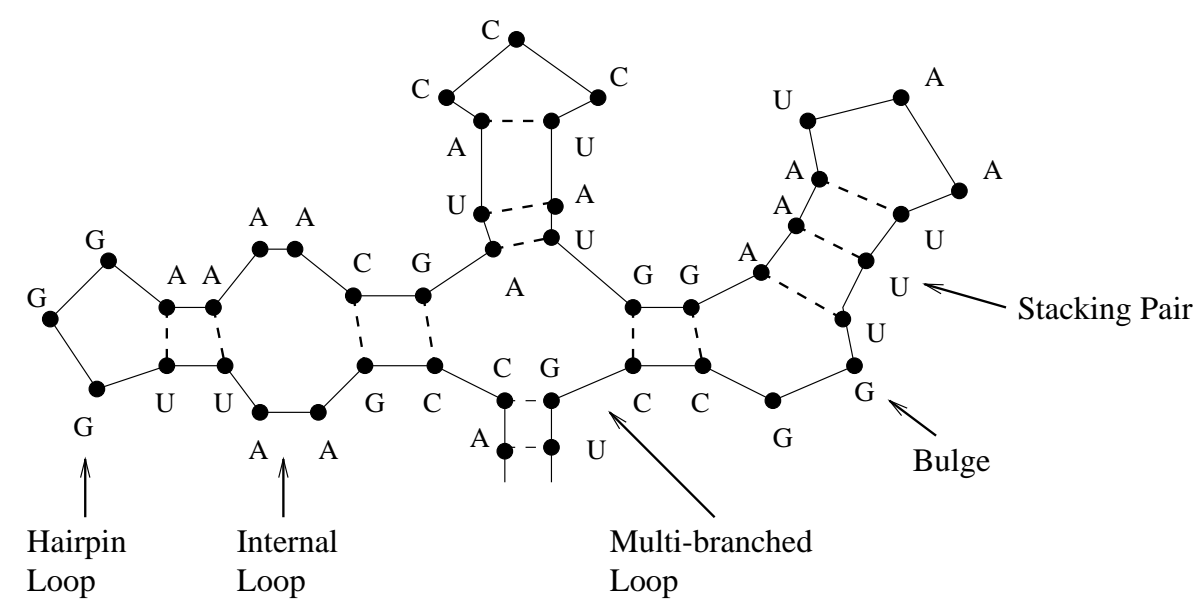

Figure 1: Example of a folded RNA

The 3-dimensional structure is related to the function of the RNA. Yet existing experimental techniques for determining the 3-dimensional structures of RNAs are often very costly and time consuming (see, e.g., [6]). The secondary structure of an RNA is the set of base pairings formed in its 3-dimensional structure. To determine the 3-dimensional structure of a given RNA sequence, it is useful to determine the corresponding secondary structure. As a result, it is important to design efficient algorithms to predict the secondary structure with computers.

From a computational viewpoint, the challenge of the RNA secondary structure prediction problem arises from some special structures called pseudoknots, which are defined as follows. Let $S$ be an RNA sequence $s_{1}, s_{2}, \cdots, s_{n}$. A pseudoknot is composed of two interleaving base pairs, i.e., $\left(s_{i}, s_{j}\right)$ and $\left(s_{k}, s_{\ell}\right)$ such that $i<k<j<\ell$. See Figure 2 for examples.

If we assume that the secondary structure of an RNA contains no pseudoknots, the secondary structure can be decomposed into a few types of loops: stacking pairs, hairpins, bulges, internal loops, and multiple loops (see, e.g., Tompa's lecture notes [9] or Waterman's book [11). A stacking pair is a loop formed by two pairs of consecutive bases $\left(s_{i}, s_{j}\right)$ and $\left(s_{i+1}, s_{j-1}\right)$ with $i+4 \leq j$. See Figure 1 for an example. By definition, a stacking pair contains no unpaired bases and any other kinds of loops contain one or more unpaired bases. Since unpaired bases are destabilizing and have positive free energy, stacking pairs are the only type of loops that have negative free energy and stabilize the secondary structure. It is also natural to assume that the free energies of loops are independent. Then an optimal pseudoknot-free secondary structure can be computed using dynamic programming in $O\left(n^{3}\right)$ time [3, 5, 12, 13].

However, pseudoknots are known to exist in some RNAs. For predicting secondary structures with pseudoknots, Nussinov et al. [7] have studied the case where the energy function is minimized when the number of base pairs is maximized and have obtained an $O\left(n^{3}\right)$-time algorithm for predicting secondary structures. Based on some special energy functions, Lyngso and Pedersen [4] have proven that determining the optimal secondary 

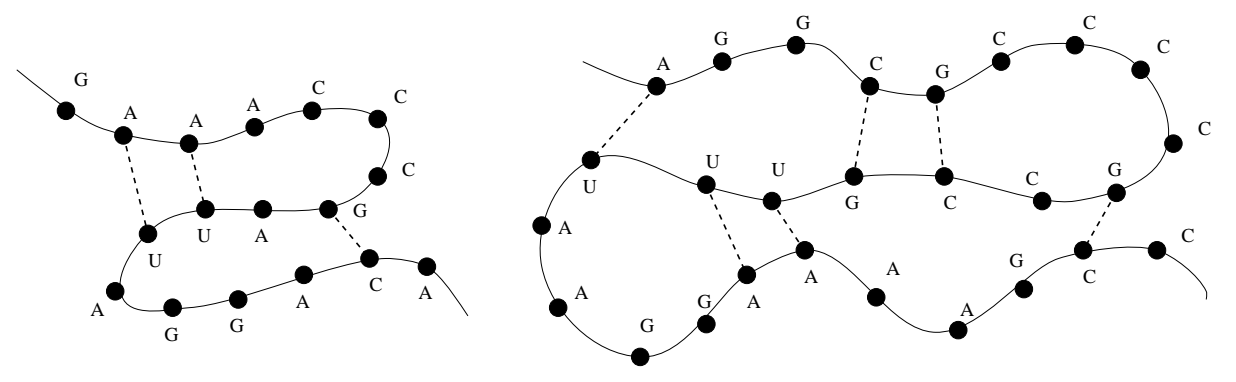

Figure 2: Examples of pseudoknots

structure possibly with pseudoknots is NP-hard. Akutsu [1] has shown that it is NP-hard to determine an optimal planar secondary structure, where a secondary structure is planar if the graph formed by the base pairings and the backbone connections of adjacent bases is planar (see Section 2 for a more detailed definition). Rivas and Eddy [8], Uemura et al. [10], and Akutsu [1] have also proposed polynomial-time algorithms that can handle limited types of pseudoknots; note that the exact types of such pseudoknots are implicit in these algorithms and difficult to determine.

Although it might be desirable to have a better classification of pseudoknots and better algorithms that can handle a wider class of pseudoknots, this paper approaches the problem in a different general direction. We initiate the study of predicting RNA secondary structures that allow arbitrary pseudoknots while maximizing the number of stacking pairs. Such a simple energy function is meaningful as stacking pairs are the only loops that stabilize secondary structures. We obtain two approximation algorithms with worst-case ratios of $1 / 2$ and $1 / 3$ for planar and general secondary structures, respectively. The planar approximation algorithm makes use of a geometric observation that allows us to visualize the planarity of stacking pairs on a rectangular grid; interestingly, such an observation does not hold if our aim is to maximize the number of base pairs. This algorithm runs in $O\left(n^{3}\right)$ time. The second approximation algorithm is more complicated and is based on a combination of multiple "greedy" strategies. A straightforward analysis cannot lead to the approximation ratio of $1 / 3$. We make use of amortization over different steps to obtain the desired ratio. This algorithm runs in $O(n)$ time.

To complement these two algorithms, we also prove that allowing pseudoknots makes it NP-hard to find the planar secondary structure with the largest number of stacking pairs. The proof makes use of a reduction from a well-known NP-complete problem called Tripartite Matching [2]. This result indicates that the hardness of the RNA secondary structure prediction problem may be inherent in the pseudoknot structures and may not be necessarily due to the complication of the energy functions. This is in contrast to the other NP-hardness results discussed earlier.

The rest of this paper is organized into four sections. Section 2 discusses some basic properties. Sections 3 and 4 present the approximation algorithms for planar and general secondary structures, respectively. Section 5 details the NP-hardness result. Section 6 concludes the paper with open problems. 


\section{Preliminaries}

Let $S=s_{1} s_{2} \cdots s_{n}$ be an RNA sequence of $n$ bases. A secondary structure $\mathcal{P}$ of $S$ is a set of Watson-Crick pairs $\left(s_{i_{1}}, s_{j_{1}}\right), \ldots,\left(s_{i_{p}}, s_{j_{p}}\right)$, where $s_{i_{r}}+2 \leq s_{j_{r}}$ for all $r=1, \ldots, p$ and no two pairs share a base. We denote $q(q \geq 1)$ consecutive stacking pairs $\left(s_{i}, s_{j}\right),\left(s_{i+1}, s_{j-1}\right)$; $\left(s_{i+1}, s_{j-1}\right), \quad\left(s_{i+2}, s_{j-2}\right) \ldots\left(s_{i+q-1}, s_{j-q+1}\right), \quad\left(s_{i+q}, s_{j-q}\right)$ of $\mathcal{P}$ by $\left(s_{i}, s_{i+1}, \ldots, s_{i+q}\right.$; $\left.s_{j-q}, \ldots, s_{j-1}, s_{j}\right)$.

Definition 1 Given a secondary structure $\mathcal{P}$, we define an undirected graph $G(\mathcal{P})$ such that the bases of $S$ are the nodes of $G(\mathcal{P})$ and $\left(s_{i}, s_{j}\right)$ is an edge of $G(\mathcal{P})$ if $j=i+1$ or $\left(s_{i}, s_{j}\right)$ is a base pair in $\mathcal{P}$.

Definition 2 A secondary structure $\mathcal{P}$ is planar if $G(\mathcal{P})$ is a planar graph.

Definition 3 A secondary structure $\mathcal{P}$ is said to contain an interleaving block if $\mathcal{P}$ contains three stacking pairs $\left(s_{i}, s_{i+1} ; s_{j-1}, s_{j}\right),\left(s_{i^{\prime}}, s_{i^{\prime}+1} ; s_{j^{\prime}-1}, s_{j^{\prime}}\right),\left(s_{i^{\prime \prime}}, s_{i^{\prime \prime}+1} ; s_{j^{\prime \prime}-1}, s_{j^{\prime \prime}}\right)$ where $i<$ $i^{\prime}<i^{\prime \prime}<j<j^{\prime}<j^{\prime \prime}$.

Lemma 2.1 If a secondary structure $\mathcal{P}$ contains an interleaving block, $\mathcal{P}$ is non-planar.

Proof. Suppose $\mathcal{P}$ contains an interleaving block. Without loss of generality, we assume that $\mathcal{P}$ contains the stacking pairs $\left(s_{1}, s_{2} ; s_{7}, s_{8}\right),\left(s_{3}, s_{4} ; s_{9}, s_{10}\right)$, and $\left(s_{5}, s_{6} ; s_{11}, s_{12}\right)$. Figure 3(a) shows the subgraph of $G(\mathcal{P})$ corresponding to these stacking pairs. Since this subgraph contains a homeomorphic copy of $K_{3,3}$ (see Figure $3(\mathrm{~b})$ ), $G(\mathcal{P})$ and $\mathcal{P}$ are non-planar.
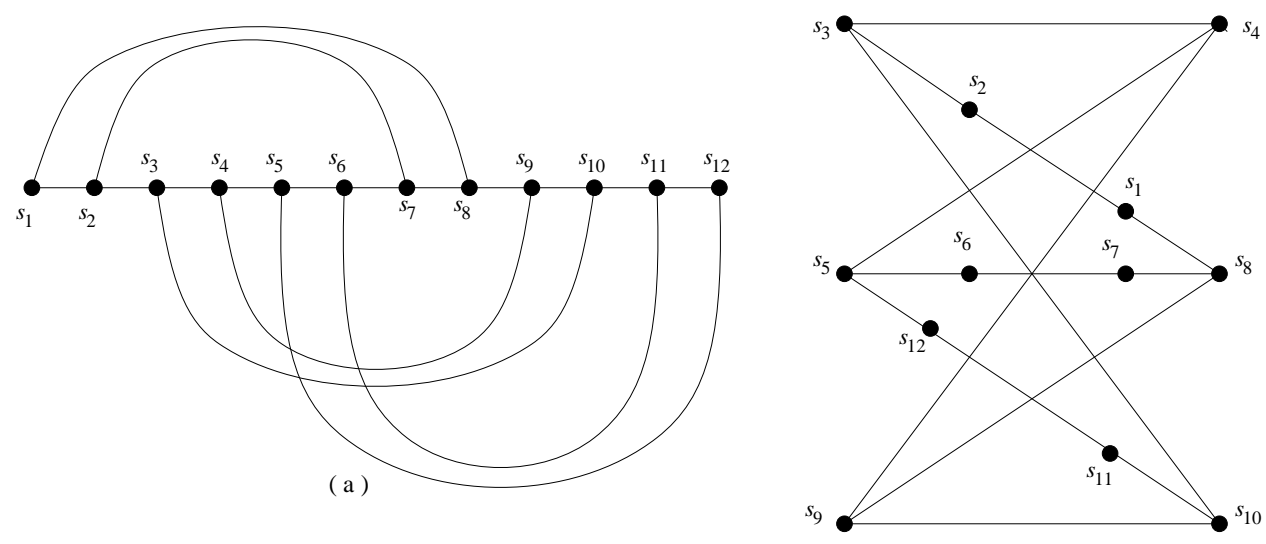

( b )

Figure 3: Interleaving block 


\section{An Approximation Algorithm for Planar Secondary Struc- tures}

We present an algorithm which, given an RNA sequence $S=s_{1} s_{2} \ldots s_{n}$, constructs a planar secondary structure of $S$ to approximate one with the maximum number of stacking pairs with a ratio of at least $1 / 2$. This approximation algorithm is based on the subtle observation in Lemma 3.1 that if a secondary structure $\mathcal{P}$ is planar, the subgraph of $G(\mathcal{P})$ which contains only the stacking pairs of $\mathcal{P}$ can be embedded in a grid with a useful property. This property enables us to consider only the secondary structure of $S$ without pseudoknots in order to achieve $1 / 2$ approximation ratio.

Definition 4 Given a secondary structure $\mathcal{P}$, we define a stacking pair embedding of $\mathcal{P}$ on a grid as follows. Represent the bases of $S$ as $n$ consecutive grid points on the same horizontal grid line $L$ such that $s_{i}$ and $s_{i+1}(1 \leq i<n)$ are connected directly by a horizontal grid edge. If $\left(s_{i}, s_{i+1} ; s_{j-1}, s_{j}\right)$ is a stacking pair of $\mathcal{P}, s_{i}$ and $s_{i+1}$ are connected to $s_{j}$ and $s_{j-1}$ respectively by a sequence of grid edges such that the two sequences must be either both above or both below $L$.

Figure 4 shows a stacking pair embedding (Figure 4(b)) of a given secondary structure (Figure $4(\mathrm{a}))$. Note that $\left(s_{3}, s_{9}\right)$ do not form a stacking pair with other base pair, so $s_{3}$ is not connected to $s_{9}$ in the stacking pair embedding. Similarly, $s_{4}$ is not connected to $s_{10}$ in the embedding.

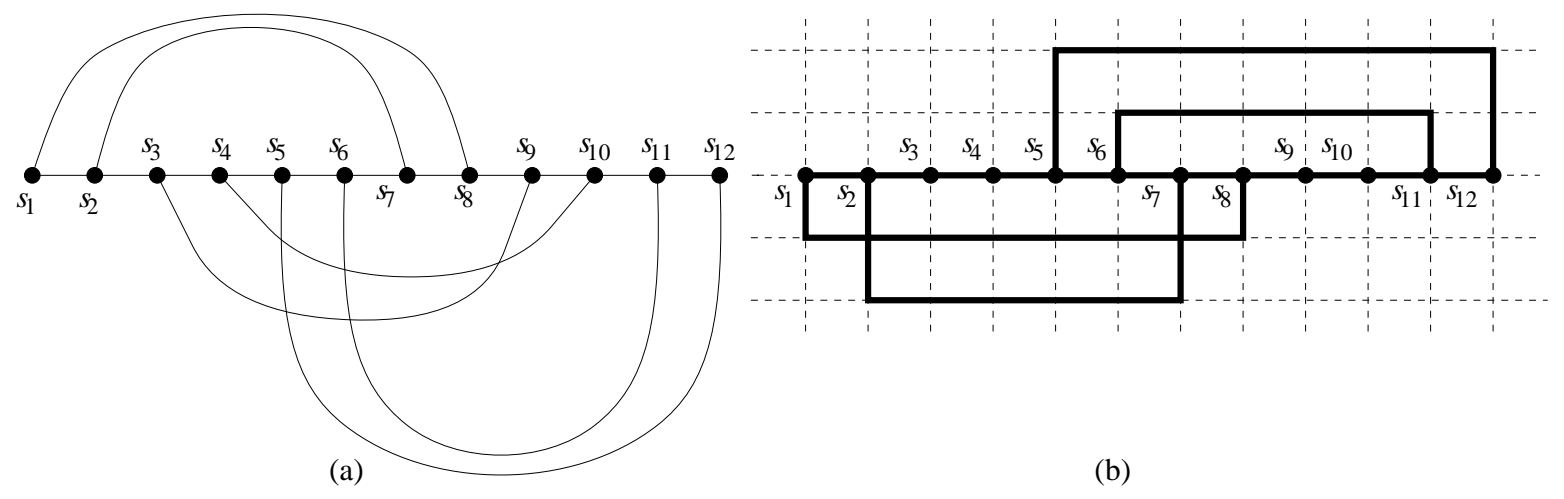

Figure 4: An example of a stacking pair embedding

Definition 5 A stacking pair embedding is said to be planar if it can be drawn in such a way that no lines cross or overlap with each other in the grid.

The embedding shown in Figure $4(\mathrm{~b})$ is planar.

Lemma 3.1 Let $\mathcal{P}$ be a secondary structure of an $R N A$ sequence $S$. Let $E$ be a stacking pair embedding of $\mathcal{P}$. If $\mathcal{P}$ is planar, then $E$ must be planar. 
Proof. If $\mathcal{P}$ does not have a planar stacking pair embedding, we claim that $\mathcal{P}$ contains an interleaving block. Let $L$ be the horizontal grid line that contains the bases of $S$ in $E$. Since $\mathcal{P}$ does not have a planar stacking pair embedding, we can assume that $E$ has two stacking pairs intersect above $L$ (see Figure $5(\mathrm{a})$ ).

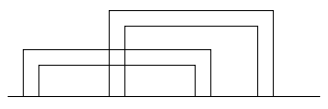

(a)

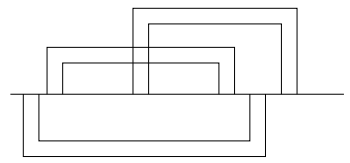

(d)

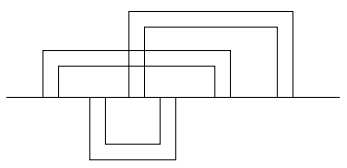

(g)

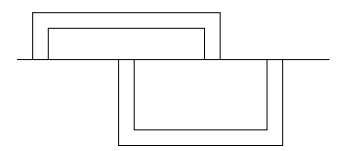

(b)

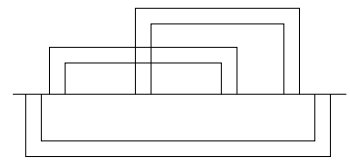

(e)

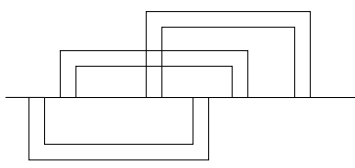

(h)

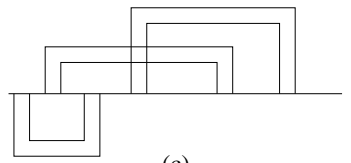

(c)

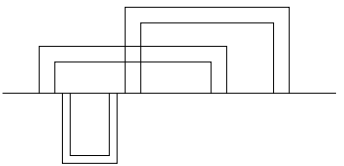

(f)

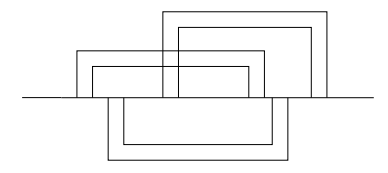

(i)

Figure 5: Non-planar stacking pair embedding

If there is no other stacking pair underneath these two pairs, we can flip one of the pairs below $L$ as shown in Figure 5(b). So, there must be at least one stacking pair underneath these two pairs. By checking all possible cases (all non-symmetric cases are shown in Figures 5(c) to (i)), it can be shown that $E$ cannot be redrawn without crossing or overlapping lines only if it contains an interleaving block (Figures $5(\mathrm{~h})$ and (i)). So, by Lemma 2.1, $\mathcal{P}$ is non-planar.

By Lemma 3.1, we can relate two secondary structures having the maximum number of stacking pairs with and without pseudoknots in the following lemma.

Lemma 3.2 Given an $R N A$ sequence $S$, let $N^{*}$ be the maximum number of stacking pairs that can be formed by a planar secondary structure of $S$ and let $W$ be the maximum number of stacking pairs that can be formed by $S$ without pseudoknots. Then, $W \geq \frac{N^{*}}{2}$.

Proof. Let $\mathcal{P}^{*}$ be a planar secondary structure of $S$ with $N^{*}$ stacking pairs. Since $\mathcal{P}^{*}$ is planar, by Lemma 3.1, any stacking pair embedding of $\mathcal{P}^{*}$ is planar.

Let $E$ be a stacking pair embedding of $\mathcal{P}^{*}$ such that no lines cross each other in the grid. Let $L$ be the horizontal grid line of $E$ which contains all bases of $S$. Let $n_{1}$ and $n_{2}$ be the number of stacking pairs which are drawn above and below $L$, respectively. Without loss of generality, assume that $n_{1} \geq n_{2}$. Now, we construct another planar secondary structure $\mathcal{P}$ from $E$ by deleting all stacking pairs which are drawn below $L$. Obviously, $\mathcal{P}$ is a planar secondary structure of $S$ without pseudoknots. Since $n_{1} \geq n_{2}, n_{1} \geq \frac{N^{*}}{2}$. As $W \geq n_{1}$, $W \geq \frac{N^{*}}{2}$. 
Based on Lemma 3.2, we now present the dynamic programming algorithm MaxSP which computes the maximium number of stacking pairs that can be formed by an RNA sequence $S=s_{1} s_{2} \ldots s_{n}$ without pseudoknots.

\section{Algorithm $\operatorname{Max} S P$}

Define $V(i, j)$ (for $j \geq i$ ) as the maximum number of stacking pairs without pseudoknots that can be formed by $s_{i} \ldots s_{j}$ if $s_{i}$ and $s_{j}$ form a Watson-Crick pair. Let $W(i, j)(j \geq i)$ be the maximum number of stacking pairs without pseudoknots that can be formed by $s_{i} \ldots s_{j}$. Obviously, $W(1, n)$ gives the maximum number of stacking pairs that can be formed by $S$ without pseudoknots.

Basis:

For $j=i, i+1, i+2$ or $i+3(j \leq n)$,

$$
\begin{aligned}
& V(i, j)=0 \quad \text { if } s_{i}, s_{j} \text { form a Watson-Crick pair; } \\
& W(i, j)=0 .
\end{aligned}
$$

Recurrence:

For $j>i+3$,

$$
\begin{aligned}
& W(i, j)=\max \left\{\begin{array}{l}
V(i, j) \\
W(i+1, j) \\
W(i, j-1)
\end{array} \text { if } s_{i}, s_{j} \text { form a Watson-Crick pair }\right\} ; \\
& V(i, j)=\max \left\{\begin{array}{l}
V(i+1, j-1)+1 \quad \text { if } s_{i+1}, s_{j-1} \text { form a Watson-Crick pair } \\
\max _{i+1 \leq k \leq j-2}\{W(i+1, k)+W(k+1, j-1)\}
\end{array}\right\} .
\end{aligned}
$$

Lemma 3.3 Given an RNA sequence $S$ of length n, Algorithm MaxSP computes the maximum number of stacking pairs that can be formed by $S$ without pseudoknots in $O\left(n^{3}\right)$ time and $O\left(n^{2}\right)$ space.

Proof. There are $O\left(n^{2}\right)$ entries $V(i, j)$ and $W(i, j)$ to be filled. To fill an entry of $V(i, j)$, we check at most $O(n)$ values. To fill an entry of $W(i, j), O(1)$ time suffices. The total time complexity for filling all entries is $O\left(n^{3}\right)$. Storing all entries requires $O\left(n^{2}\right)$ space.

Although Algorithm MaxSP presented in the above only computes the number of stacking pairs, it can be easily modified to compute the secondary structure. Thus we have the following theorem.

Theorem 3.4 The Algorithm MaxSP is an (1/2)-approximation algorithm for the problem of constructing a secondary structure which maximizes the number of stacking pairs for an $R N A$ sequence $S$. 


\section{An Approximation Algorithm for General Secondary Struc- tures}

We present Algorithm Greedy $S P()$ which, given an RNA sequence $S=s_{1} s_{2} \ldots s_{n}$, constructs a secondary structure of $S$ (not necessarily planar) with at least $1 / 3$ of the maximum possible number of stacking pairs. The approximation algorithm uses a greedy approach. Figure 6 shows the algorithm Greedy $S P()$.

// Let $S=s_{1} s_{2} \ldots s_{n}$ be the input RNA sequence. Initially, all $s_{j}$ are unmarked.

// Let $E$ be the set of base pairs output by the algorithm. Initially, $E=\emptyset$.

$\operatorname{Greedy} S P(S, i) \quad / / i \geq 3$

1. Repeatedly find the leftmost $i$ consecutive stacking pairs $S P$ (i.e., find $\left(s_{p}, \ldots, s_{p+i} ; s_{q-i}, \ldots, s_{q}\right)$ such that $p$ is as small as possible) formed by unmarked bases. Add $S P$ to $E$ and mark all these bases.

2. For $k=i-1$ downto 2 ,

Repeatedly find any $k$ consecutive stacking pairs $S P$ formed by unmarked bases. Add $S P$ to $E$ and mark all these bases.

3. Repeatedly find the leftmost stacking pair $S P$ formed by unmarked bases. Add $S P$ to $E$ and mark all these bases.

Figure 6: A 1/3-Approximation Algorithm

In the following, we analyze the approximation ratio of this algorithm. The algorithm $\operatorname{Greedy} S P(S, i)$ will generate a sequence of $S P$ 's denoted by $S P_{1}, S P_{2}, \ldots, S P_{h}$.

Fact 4.1 For any $S P_{j}$ and $S P_{k}(j \neq k)$, the stacking pairs in $S P_{j}$ do not share any base with those in $S P_{k}$.

For each $S P_{j}=\left(s_{p}, \ldots, s_{p+t} ; s_{q-t}, \ldots, s_{q}\right)$, we define two intervals of indexes, $\mathcal{I}_{j}$ and $\mathcal{J}_{j}$, as $[p . . p+t]$ and $[q-t . . q]$, respectively. In order to compare the number of stacking pairs formed with that in the optimal case, we have the following definition.

Definition 6 Let $\mathcal{P}$ be an optimal secondary structure of $S$ with the maximum number of stacking pairs. Let $\mathcal{F}$ be the set of all stacking pairs of $\mathcal{P}$. For each $S P_{j}$ computed by GreedySP $(S, i)$ and $\beta=\mathcal{I}_{j}$ or $\mathcal{J}_{j}$,

let $\mathcal{X}_{\beta}=\left\{\left(s_{k}, s_{k+1} ; s_{w-1}, s_{w}\right) \in \mathcal{F} \mid\right.$ at least one of indexes $k, k+1, w-1, w$ is in $\left.\beta\right\}$.

Note that $\mathcal{X}_{\beta}$ 's may not be disjoint.

Lemma 4.2 $\bigcup_{1 \leq j \leq h}\left\{\mathcal{X}_{\mathcal{I}_{j}} \cup \mathcal{X}_{\mathcal{J}_{j}}\right\}=\mathcal{F}$.

Proof. We prove this lemma by contradiction. Suppose that there exists a stacking pair $\left(s_{k}, s_{k+1} ; s_{w-1}, s_{w}\right)$ in $\mathcal{F}$ but not in any of $\mathcal{X}_{\mathcal{I}_{j}}$ and $\mathcal{X}_{\mathcal{J}_{j}}$. By Definition 6 , none of the 
indexes, $k, k+1, w-1, w$ is in any of $\mathcal{I}_{j}$ and $\mathcal{J}_{j}$. This contradicts with Step 3 of Algorithm GreedySP $(S, i)$.

Definition 7 For each $\mathcal{X}_{\mathcal{I}_{j}}$,

$$
\text { let } \mathcal{X}_{\mathcal{I}_{j}}^{\prime}=\mathcal{X}_{\mathcal{I}_{j}}-\bigcup_{k<j}\left\{\mathcal{X}_{\mathcal{I}_{k}} \cup \mathcal{X}_{\mathcal{J}_{k}}\right\} \text {, and let } \mathcal{X}_{\mathcal{J}_{j}}^{\prime}=\mathcal{X}_{\mathcal{J}_{j}}-\bigcup_{k<j}\left\{\mathcal{X}_{\mathcal{I}_{k}} \cup \mathcal{X}_{\mathcal{J}_{k}}\right\}-\mathcal{X}_{\mathcal{I}_{j}}
$$

Let $\left|S P_{j}\right|$ be the number of stacking pairs represented by $S P_{j}$. Let $\left|\mathcal{I}_{j}\right|$ and $\left|\mathcal{J}_{j}\right|$ be the numbers of indexes in the intervals $\mathcal{I}_{j}$ and $\mathcal{J}_{j}$, respectively.

Lemma 4.3 Let $N$ be the number of stacking pairs computed by Algorithm GreedySP $(S, i)$ and $N^{*}$ be the maximum number of stacking pairs that can be formed by $S$. If for all $j$, we have $\left|S P_{j}\right| \geq \frac{1}{r} \times\left|\left(\mathcal{X}_{\mathcal{I}_{j}}^{\prime} \cup \mathcal{X}_{\mathcal{J}_{j}}^{\prime}\right)\right|$, then $N \geq \frac{1}{r} \times N^{*}$.

Proof. By Definition [7, $\bigcup_{k}\left\{\mathcal{X}_{\mathcal{I}_{k}} \cup \mathcal{X}_{\mathcal{J}_{k}}\right\}=\bigcup_{k}\left\{\mathcal{X}_{\mathcal{I}_{k}}^{\prime} \cup \mathcal{X}_{\mathcal{J}_{k}}^{\prime}\right\}$. Then by Fact 4.1, $N=$ $\sum_{j}\left|S P_{j}\right|$. Thus, $N \geq \frac{1}{r} \times\left|\bigcup_{k}\left\{\mathcal{X}_{\mathcal{I}_{k}} \cup \mathcal{X}_{\mathcal{J}_{k}}\right\}\right|$. By Lemma 4.2, $N \geq \frac{1}{r} \times N^{*}$.

Lemma 4.4 For each $S P_{j}$ computed by $\operatorname{Greedy} S P(S, i)$, we have $\left|S P_{j}\right| \geq \frac{1}{3} \times\left|\left(\mathcal{X}_{\mathcal{I}_{j}}^{\prime} \cup \mathcal{X}_{\mathcal{J}_{j}}^{\prime}\right)\right|$.

Proof. There are three cases as follows.

Case 1: $S P_{j}$ is computed by $\operatorname{Greedy} S P(S, i)$ in Step 1. Note that $S P_{j}=\left(s_{p}, \ldots, s_{p+i}\right.$; $\left.s_{q-i}, \ldots, s_{q}\right)$ is the leftmost $i$ consecutive stacking pairs, i.e., $p$ is the smallest possible. By definition, $\left|\mathcal{X}_{\mathcal{I}_{j}}^{\prime}\right|,\left|\mathcal{X}_{\mathcal{J}_{j}}^{\prime}\right| \leq i+2$. We further claim that $\left|\mathcal{X}_{\mathcal{I}_{j}}^{\prime}\right| \leq i+1$. Then $\left|S P_{j}\right| /\left|\mathcal{X}_{\mathcal{I}_{j}}^{\prime} \cup \mathcal{X}_{\mathcal{J}_{j}}^{\prime}\right| \geq$ $i /((i+1)+(i+2)) \geq 1 / 3($ as $i \geq 3)$.

We prove the claim by contradiction. Assume that $\left|\mathcal{X}_{\mathcal{I}_{j}}^{\prime}\right|=i+2$. That is, for some integer $t, \mathcal{F}$ has $i+2$ consecutive stacking pairs $\left(s_{p-1}, \ldots, s_{p+i+1} ; s_{t-i-1}, \ldots, s_{t+1}\right)$. Furthermore, none of the bases $s_{p-1}, \ldots, s_{p+i+1}, s_{t-i-1}, \ldots, s_{t+1}$ are marked before $S P_{j}$ is chosen; otherwise, suppose one such base, says $s_{a}$, is marked when the algorithm chooses $S P_{\ell}$ for $\ell<j$, then an stacking pair adjacent to $s_{a}$ does not belong to $\mathcal{X}_{\mathcal{I}_{j}}^{\prime}$ and they belong to $\mathcal{X}_{\mathcal{I}_{\ell}}^{\prime}$ or $\mathcal{X}_{\mathcal{J}_{\ell}}^{\prime}$ instead. Therefore, $\left(s_{p-1}, \ldots, s_{p+i-1} ; s_{t-i+1}, \ldots, s_{t+1}\right)$ is the leftmost $i$ consecutive stacking pairs formed by unmarked bases before $S P_{j}$ is chosen. As $S P_{j}$ is not the leftmost $i$ consecutive stacking pairs, this contradicts the selection criteria of $S P_{j}$. The claim follows.

Case 2: $S P_{j}$ is computed by $\operatorname{Greedy} S P(S, i)$ in Step 2. Let $\left|S P_{j}\right|=k \geq 2$. Let $S P_{j}=$ $\left(s_{p}, \ldots, s_{p+k} ; s_{q-k}, \ldots, s_{q}\right)$. By definition, $\left|\mathcal{X}_{\mathcal{I}_{j}}^{\prime}\right|,\left|\mathcal{X}_{\mathcal{J}_{j}}^{\prime}\right| \leq k+2$. We claim that $\left|\mathcal{X}_{\mathcal{I}_{j}}^{\prime}\right|,\left|\mathcal{X}_{\mathcal{J}_{j}}^{\prime}\right| \leq$ $k+1$. Then $\left|S P_{j}\right| /\left|\mathcal{X}_{\mathcal{I}_{j}}^{\prime} \cup \mathcal{X}_{\mathcal{J}_{j}}^{\prime}\right| \geq k /((k+1)+(k+1))$, which is at least $1 / 3$ as $k \geq 2$.

To show that $\left|\mathcal{X}_{\mathcal{I}_{j}}^{\prime}\right| \leq k+1$ by contradiction, assume $\left|\mathcal{X}_{\mathcal{I}_{j}}^{\prime}\right|=k+2$. Thus, for some integer $t$, there exist $k+2$ consecutive stacking pairs $\left(s_{p-1}, \ldots, s_{p+k+1} ; s_{t-k-1}, \ldots, s_{t+1}\right)$. Similarly to case 1 , we can show that none of the bases $s_{p-1}, \ldots, s_{p+k+1}, s_{t-k-1}, \ldots, s_{t+1}$ are marked before $S P_{j}$ is chosen. Thus, $\operatorname{Greedy} S P(S, i)$ should select some $k+1$ or $k+2$ consecutive stacking pairs instead of the chosen $k$ consecutive stacking pairs, reaching a contradiction. Similarly, we can show $\left|\mathcal{X}_{\mathcal{J}_{j}}^{\prime}\right| \leq k+1$.

Case 3: $S P_{j}$ is computed by $\operatorname{Greedy} S P(S, i)$ in Step 3. $S P_{j}$ is the leftmost stacking pair when it is chosen. Let $S P_{j}=\left(s_{p}, s_{p+1} ; s_{q-1}, s_{q}\right)$. By the same approach as in Case 2, we can show $\left|\mathcal{X}_{\mathcal{I}_{j}}^{\prime}\right|,\left|\mathcal{X}_{\mathcal{J}_{j}}^{\prime}\right| \leq 2$. We further claim $\left|\mathcal{X}_{\mathcal{I}_{j}}^{\prime}\right| \leq 1$. Then $\left|S P_{j}\right| /\left|\mathcal{X}_{\mathcal{I}_{j}}^{\prime} \cup \mathcal{X}_{\mathcal{J}_{j}}^{\prime}\right| \geq 1 /(1+2)=1 / 3$. 
To verify $\left|\mathcal{X}_{\mathcal{I}_{j}}^{\prime}\right| \leq 1$, we consider all possible cases with $\left|\mathcal{X}_{\mathcal{I}_{j}}^{\prime}\right|=2$ while there are no two consecutive stacking pairs. The only possible case is that for some integers $r, t$, both $\left(s_{p-1}, s_{p} ; s_{r-1}, s_{r}\right)$ and $\left(s_{p}, s_{p+1} ; s_{t-1}, s_{t}\right)$ belong to $\mathcal{X}_{\mathcal{I}_{j}}^{\prime}$. Then, $S P_{j}$ cannot be the leftmost stacking pair formed by unmarked bases, contradicting the selection criteria of $S P_{j}$.

Theorem 4.5 Let $S$ be an $R N A$ sequence. Let $N^{*}$ be the maximum number of stacking pairs that can be formed by any secondary structure of $S$. Let $N$ be the number of stacking pairs output by Greedy $S P(S, i)$. Then, $N \geq \frac{N^{*}}{3}$.

Proof. By Lemmas 4.3 and 4.4 , the result follows.

We remark that by setting $i=3$ in $\operatorname{Greedy} S P(S, i)$, we can already achieve the approximation ratio of $1 / 3$. The following theorem gives the time and space complexity of the algorithm.

Theorem 4.6 Given an RNA sequence $S$ of length $n$ and a constant $k$, Algorithm Greedy $S P(S, k)$ can be implemented in $O(n)$ time and $O(n)$ space.

Proof. Recall that the bases of an RNA sequence are chosen from the alphabet $\{A, U, G, C\}$. If $k$ is a constant, there are only constant number of different patterns of consecutive stacking pairs that we must consider. For any $1 \leq j \leq k$, there are only $4^{j}$ different strings that can be formed by the four characters $\{A, U, G, C\}$. So, the locations of the occurrences of these possible strings in the RNA sequence can be recorded in an array of linked lists indexed by the pattern of the string using $O(n)$ time preprocessing. There are at most $4^{j}$ linked lists for any fixed $j$ and there are at most $n$ entries in these linked lists. In total, there are at most $k n$ entries in all linked lists for all possible values of $j$.

Now, we fix a constant $j$. To locate all $j$ consecutive stacking pairs, we scan the RNA sequence from left to right. For each substring of $j$ consecutive characters, we look up the array to see whether we can form $j$ consecutive stacking pairs. By simple bookkeeping, we can keep track which bases have been used already. Each entry in the linked lists will only be scanned at most once, so the whole procedure takes only $O(n)$ time. Since $k$ is a constant, we can repeat the whole procedure for $k$ different values of $j$, and the total time complexity is still $O(n)$ time.

\section{$5 \quad$ NP-completeness}

In this section, we show that it is NP-hard to find a planar secondary structure with the largest number of stacking pairs. We consider the following decision problem. Given an RNA sequence $S$ and an integer $h$, we wish to determine whether the largest possible number of stacking pairs in a planar secondary structure of $S$, denoted $\operatorname{sp}(S)$, is at least $h$. Below we show that this decision problem is NP-complete by reducing the tripartite matching problem [2] to it, which is defined as follows.

Given three node sets $X, Y$, and $Z$ with the same cardinality $n$ and an edge set $E \subseteq$ $X \times Y \times Z$ of size $m$, the tripartite matching problem is to determine whether $E$ contains 
a perfect matching, i.e., a set of $n$ edges which touches every node of $X, Y$, and $Z$ exactly once.

The remainder of this section is organized as follows. Section 5.1 shows how we construct in polynomial time an RNA sequence $S_{E}$ and an integer $h$ from a given instance $(X, Y, Z, E)$ of the tripartite matching problem, where $h$ depends on $n$ and $m$. Section 5.2 shows that if $E$ contains a perfect matching, then $\operatorname{sp}\left(S_{E}\right) \geq h$. Section 5.3 is the non-trivial part, showing that if $E$ does not contain a perfect matching, then $\operatorname{sp}\left(S_{E}\right)<h$. Combining these three sections, we can conclude that it is NP-hard to maximize the number of stacking pairs for planar RNA secondary structures.

\subsection{Construction of the RNA sequence $S_{E}$}

Consider any instance $(X, Y, Z, E)$ of the tripartite matching problem. We construct an RNA sequence $S_{E}$ and an integer $h$ as follows. Let $X=\left\{x_{1}, \cdots, x_{n}\right\}, Y=\left\{y_{1}, \cdots, y_{n}\right\}$, and $Z=\left\{z_{1}, \cdots, z_{n}\right\}$. Furthermore, let $E=\left\{e_{1}, e_{2}, \cdots, e_{m}\right\}$, where each edge $e_{j}=$ $\left(x_{p_{j}}, y_{q_{j}}, z_{r_{j}}\right)$. Recall that an RNA sequence contains characters chosen from the alphabet $\{A, U, G, C\}$. Below we denote $A^{i}$, where $i$ is any positive integer, as the sequence of $i A^{\prime}$ 's. Furthermore, $A^{+}$means a sequence of one or more $A$ 's.

Let $d=\max \{6 n, 4(m+1)\}+1$. Define the following four RNA sequences for every positive integer $k<d$.

- $\delta(k)$ is the sequence $U^{d} A^{k} G U^{d} A^{d-k}$, and $\overline{\delta(k)}$ is the sequence $U^{d-k} A^{d} G U^{k} A^{d}$.

- $\pi(k)$ is the sequence $C^{2 d+2 k} A G C^{4 d-2 k}$, and $\overline{\pi(k)}$ is the sequence $G^{4 d-2 k} A G^{2 d+2 k}$.

Fragments: Note that the sequences $\delta(k)$ and $\overline{\delta(k)}$ are each composed of two substrings in the form of $U^{+} A^{+}$, separated by a character $G$. Each of these two substrings is called a fragment. Similarly, the two substrings of the form $C^{+}$separated by $A G$ in $\pi(k)$ and the two substrings of the form $G^{+}$separated by the character $A$ in $\overline{\pi(k)}$ are also called fragments.

Node Encoding: Each node in the three node sets $X, Y$, and $Z$ is associated with a unique sequence. For $1 \leq i \leq n$, let $\left\langle x_{i}\right\rangle,\left\langle y_{i}\right\rangle,\left\langle z_{i}\right\rangle$ denote the sequences $\delta(i), \delta(n+i)$, $\delta(2 n+i)$, respectively. Intuitively, $\left\langle x_{i}\right\rangle$ is the encoding of the node $x_{i}$, and similarly $\left\langle y_{i}\right\rangle$ and $\left\langle z_{i}\right\rangle$ are for the nodes $y_{i}$ and $z_{i}$, respectively. Furthermore, define $\left\langle\overline{x_{i}}\right\rangle=\overline{\delta(i)},\left\langle\overline{y_{i}}\right\rangle=\overline{\delta(n+i)}$, and $\left\langle\overline{z_{i}}\right\rangle=\overline{\delta(2 n+i)}$.

The node set $X$ is associated with two sequences $\mathcal{X}=\left\langle x_{1}\right\rangle G\left\langle x_{2}\right\rangle G \cdots G\left\langle x_{n}\right\rangle$ and $\overline{\mathcal{X}}=$ $\left\langle\overline{x_{n}}\right\rangle G\left\langle\overline{x_{n-1}}\right\rangle G \cdots G\left\langle\overline{x_{1}}\right\rangle$. Let $\mathcal{X}-x_{i}=\left\langle x_{1}\right\rangle G \cdots G\left\langle x_{i-1}\right\rangle G\left\langle x_{i+1}\right\rangle G \cdots\left\langle x_{n}\right\rangle$ and $\overline{\mathcal{X}-x_{i}}=$ $\left\langle\overline{x_{n}}\right\rangle G \cdots G\left\langle\overline{x_{i+1}}\right\rangle G\left\langle\overline{x_{i-1}}\right\rangle G \cdots G\left\langle\overline{x_{1}}\right\rangle$, where $x_{i}$ is any node in $X$. Similarly, the node sets $Y$ and $Z$ are associated with sequences $\mathcal{Y}, \overline{\mathcal{Y}}$, and $\mathcal{Z}, \overline{\mathcal{Z}}$, respectively.

Edge Encoding: For each edge $e_{j}$ (where $1 \leq j \leq m$ ), we define four delimiter sequences, namely, $V_{j}=\pi(j), W_{j}=\pi(m+1+j), \overline{V_{j}}=\overline{\pi(j)}$, and $\overline{W_{j}}=\overline{\pi(m+1+j)}$. Assume that $e_{j}=\left(x_{p_{j}}, y_{q_{j}}, z_{r_{j}}\right)$. Then $e_{j}$ is encoded by the sequence $S_{j}$ defined as

$$
A G V_{j} A G W_{j} A G \mathcal{X} G \mathcal{Y} G \mathcal{Z} G \overline{\left(\mathcal{Z}-z_{r_{j}}\right)} G \overline{\left(\mathcal{Y}-y_{q_{j}}\right)} G \overline{\left(\mathcal{X}-x_{p_{j}}\right)} \overline{V_{j}} A \overline{W_{j}}
$$


Let $S_{m+1}$ be a special sequence defined as $A G V_{m+1} A G W_{m+1} A G \overline{\mathcal{Z}} G \overline{\mathcal{Y}} G \overline{\mathcal{X}} \overline{V_{m+1}} A \overline{W_{m+1}}$. In the following discussion, each $S_{j}$ is referred to as a region.

Finally, we define $S_{E}$ to be the sequence $S_{m+1} S_{m} \cdots S_{1}$. Let $\sigma=3 n(3 d-2)+6 d-1$ and let $h=m \sigma+n(6 d-4)+12 d-5$. Note that $S_{E}$ has $O\left((n+m)^{3}\right)$ characters and can be constructed in $O\left(\left|S_{E}\right|\right)$ time. In Sections 5.2 and 5.3, we show that $\operatorname{sp}\left(S_{E}\right) \geq h$ if and only if $E$ contains a perfect matching.

\subsection{Correctness of the if-part}

This section shows that if $E$ has a perfect matching, we can construct a planar secondary structure for $S_{E}$ containing at least $h$ stacking pairs. Therefore, $\operatorname{sp}\left(S_{E}\right) \geq h$.

First of all, we establish several basic steps for constructing stacking pairs on $S_{E}$.

- $\delta(i)$ or $\overline{\delta(i)}$ itself can form $d-1$ stacking pairs, while $\delta(i)$ and $\overline{\delta(i)}$ together can form $3 d-2$ stacking pairs.

- $\pi(i)$ and $\overline{\pi(i)}$ together can form $6 d-2$ stacking pairs.

- For any $i \neq j, \pi(i)$ and $\overline{\pi(j)}$ together can form $6 d-3$ stacking pairs.

Lemma 5.1 If E has a perfect matching, then $\operatorname{sp}\left(S_{E}\right) \geq h$.

Proof. Let $M=\left\{e_{j_{1}}, e_{j_{2}}, \ldots, e_{j_{n}}\right\}$ be a perfect matching. Without loss of generality, we assume that $1 \leq j_{1}<j_{2}<\ldots<j_{n} \leq m$. Define $j_{n+1}=m+1$. To obtain a planar secondary structure for $S_{E}$ with at least $h$ stacking pairs, we consider the regions one by one. There are three cases.

Case 1: We consider any region $S_{j}$ such that $e_{j} \notin M$. Our goal is to show that $\sigma=$ $3 n(3 d-2)+6 d-1$ stacking pairs can be formed within $S_{j}$. Note that there are $(m-n)$ edges not in $M$. Thus, we can obtain a total of $(m-n) \sigma$ stacking pairs in this case. Details are as follows. Assume that $e_{j}=\left(x_{p_{j}}, y_{q_{j}}, z_{r_{j}}\right)$.

- $6 d-2$ stacking pairs can be formed between $V_{j}$ and $\overline{V_{j}}$, and between $W_{j}$ and $\overline{W_{j}}$.

- $3 d-2$ stacking pairs can be formed between $\left\langle x_{i}\right\rangle$ and $\left\langle\overline{x_{i}}\right\rangle$ for all $i \neq p_{j}$, and between $\left\langle y_{i}\right\rangle$ and $\left\langle\overline{y_{i}}\right\rangle$ for all $i \neq q_{j}$, and between $\left\langle z_{i}\right\rangle$ and $\left\langle\overline{z_{i}}\right\rangle$ for all $i \neq r_{j}$.

- $\left\langle\overline{x_{p_{j}}}\right\rangle,\left\langle\overline{y_{q_{j}}}\right\rangle$, and $\left\langle\overline{z_{r_{j}}}\right\rangle$ can each form $d-1$ stacking pairs.

The total number of stacking pairs that can be formed within $S_{j}$ is $2(6 d-2)+3(n-1)(3 d-$ $2)+3(d-1)=3 n(3 d-2)+6 d-1=\sigma$.

Case 2: We consider the edges $e_{j_{1}}, e_{j_{2}}, \ldots, e_{j_{n}}$ in $M$. Our goal is to show that each corresponding region accounts for $\sigma+6 d-4$ stacking pairs. Thus, we obtain a total of $n \sigma+n(6 d-4)$ stacking pairs in this case. Details are as follows. Unlike Case 1, each region $S_{j_{k}}$, where $1 \leq k \leq n$, may have some of its bases paired with that of $S_{j_{k+1}}$.

- $6 d-3$ stacking pairs can be formed between $W_{j_{k}}$ in $S_{j_{k}}$ and $\overline{W_{j_{k+1}}}$ in $S_{j_{k+1}}$.

- $6 d-2$ stacking pairs can be formed between $V_{j_{k}}$ in $S_{j_{k}}$ and $\overline{V_{j_{k}}}$ in $S_{j_{k}}$. 
- $3 d-2$ stacking pairs can be paired between $\left\langle x_{i}\right\rangle$ in $S_{j_{k}}$ and $\left\langle\overline{x_{i}}\right\rangle$ in $S_{j_{k}}$ for any $i \neq p_{j_{1}}, \ldots, p_{j_{k}}$, and between $\left\langle y_{i}\right\rangle$ in $S_{j_{k}}$ and $\left\langle\overline{y_{i}}\right\rangle$ in $S_{j_{k}}$ for any $i \neq q_{j_{1}}, \ldots, q_{j_{k}}$, and between $\left\langle z_{i}\right\rangle$ in $S_{j_{k}}$ and $\left\langle\overline{z_{i}}\right\rangle$ in $S_{j_{k}}$ for any $i \neq r_{j_{1}}, \ldots, r_{j_{k}}$.

- $3 d-2$ stacking pairs can be paired between $\left\langle x_{i}\right\rangle$ in $S_{j_{k}}$ and $\left\langle\overline{x_{i}}\right\rangle$ in $S_{j_{k+1}}$ for any $i=p_{j_{1}}, \ldots, p_{j_{k}}$, and between $\left\langle y_{i}\right\rangle$ in $S_{j_{k}}$ and $\left\langle\overline{y_{i}}\right\rangle$ in $S_{j_{k+1}}$ for any $i=q_{j_{1}}, \ldots, q_{j_{k}}$, and between $\left\langle z_{i}\right\rangle$ in $S_{j_{k+1}}$ and $\left\langle\overline{z_{i}}\right\rangle$ in $S_{j_{k+1}}$ for any $i=r_{j_{1}}, \ldots, r_{j_{k}}$.

The total number of stacking pairs charged to $S_{j_{k}}$ is $6 d-3+6 d-2+3 n(3 d-2)=\sigma+6 d-4$.

Case 3: We consider $S_{m+1}$. We can form $6 d-2$ stacking pairs between $V_{m+1}$ and $\overline{V_{m+1}}$, and $6 d-3$ stacking pairs between $W_{m+1}$ and $\overline{W_{j_{1}}}$. The number of such stacking pairs is $12 d-5$.

Combining the three cases, the number of stacking pairs that can be formed on $S_{E}$ is $(m-n) \sigma+n(\sigma+6 d-4)+12 d-5$, which is exactly $h$. Notice that no two stacking pairs formed cross each other. Thus, $\operatorname{sp}\left(S_{E}\right) \geq h$.

\subsection{Correctness of the only-if part}

This section shows that if $E$ has no perfect matching, then $\operatorname{sp}\left(S_{E}\right)<h$. We first give the framework of the proof in Section 5.3.1. Then, some basic definitions and concepts are presented in Section 5.3.2. The proof of the only-if part is given in Section 5.3.3.

\subsubsection{Framework of the proof}

Let OPT be a secondary structure of $S_{E}$ with the maximum number of stacking pairs. Let \#OPT be the number of stacking pairs in OPT. That is, \#OPT $=\operatorname{sp}\left(S_{E}\right)$. In this section, we will establish an upper bound for \#OPT. Recall that we only consider Watson-Crick base pairs, i.e., $A-U$ and $C-G$ pairs. We define a conjugate of a substring in $S_{E}$ as follows.

Conjugates: For every substring $R=s_{1} s_{2} \ldots s_{k}$ of $S_{E}$, the conjugate of $R$ is $\hat{R}=\hat{s_{k}} \ldots \hat{s_{1}}$, where $\hat{A}=U, \hat{U}=A, \hat{C}=G$, and $\hat{G}=C$.

For example, $A A$ 's conjugate is $U U$ and $U A$ 's conjugate is $U A$. To form a stacking pair, two adjacent bases must be paired with another two adjacent bases. So, we concentrate on the possible patterns of adjacent bases in $S_{E}$.

2-substrings: In $S_{E}$, any two adjacent characters are referred to as a 2-substring. By construction, $S_{E}$ has only ten different types of 2-substrings: $U U, A A, U A, G G, C C, G C$, $A G, G A, G U$, and $C A$-substrings. A 2-substring can only form a stacking pair with its conjugate. If they actually form a stacking pair in $O P T$, they are said to be paired.

Since the conjugates of $A G, G A, G U$, and $C A$-substrings do not exist in $S_{E}$, there is no stacking pair in $S_{E}$ which involves these 2-substrings. We only need to consider $A A$, $U U, U A, G G, C C, G C$-substrings. Table 11 shows the numbers of occurrences of these 2-substrings in $S_{j}(1 \leq j \leq m+1)$ and the total occurrences of these substrings in $S_{E}$. 


\begin{tabular}{|l|l|l||l|}
\hline \multirow{2}{*}{$\begin{array}{l}\text { Substring } \\
(t)\end{array}$} & \multicolumn{3}{|c|}{ Total number of occurrences of $t$ in } \\
\cline { 2 - 4 } & $S_{j}(j=1,2, \ldots, m)$ & $S_{m+1}$ & $S_{E}$ \\
\hline $\mathrm{AA}$ & $3 n(d-2)+(3 n-3)(2 d-2)$ & $3 n(2 d-2)$ & $m(3 n(d-2)+(3 n-3)(2 d-2))+3 n(2 d-2)$ \\
$\mathrm{UU}$ & $3 n(2 d-2)+(3 n-3)(d-2)$ & $3 n(d-2)$ & $m(3 n(2 d-2)+(3 n-3)(d-2))+3 n(d-2)$ \\
$\mathrm{UA}$ & $2(6 n-3)$ & $6 n$ & $2 m(6 n-3)+6 n$ \\
$\mathrm{GG}$ & $2(6 d-2)$ & $2(6 d-2)$ & $2(m+1)(6 d-2)$ \\
$\mathrm{CC}$ & $2(6 d-2)$ & $2(6 d-2)$ & $2(m+1)(6 d-2)$ \\
$\mathrm{GC}$ & 4 & 4 & $4 m+4$ \\
\hline
\end{tabular}

Table 1: Number of occurrences of different 2-substrings

Let \#AA denote the number of occurrences of $A A$-substrings in $S_{E}$. We use the \# notation for other types of 2-subtrings in $S_{E}$ similarly. The following fact gives a straightforward upper bound for \#OPT.

Fact $5.2 \# \mathrm{OPT} \leq \min \{\# A A, \# U U\}+\min \{\# G G, \# C C\}+\# U A / 2+\# G C / 2$

$$
=h+n+1+(2 m+2) \text {. }
$$

Note that OPT may not pair all $A A$-subtrings with $U U$-substrings. Let $\diamond A A$ be the number of $A A$-substrings that are not paired in OPT. Again, we use the $\diamond$ notaion for other types of 2-substrings. Fact 5.2 can be strengthened as follows.

Fact $5.3 \# \mathrm{OPT} \leq \min \{\# A A-\diamond A A, \# U U-\diamond U U\}+\min \{\# G G-\diamond G G, \# C C-\diamond C C\}+$ $(\# U A-\diamond U A) / 2+(\# G C-\diamond G C) / 2$.

The upper bound given in Fact 5.3 forms the basis of our proof for showing that \#OPT < $h$. In the following sections, we consider the possible structure of OPT. For each possible case, we show that the lower bounds for some $\diamond$ values, such as $\diamond A A$ and $\diamond C C$, are sufficiently large so that OPT can be shown to be less than $h$. In particular, in one of the cases, we must make use of the fact that $E$ does not have a perfect matching in order to prove the lower bound for $\diamond A A, \diamond U A$, and $\diamond U U$. We give some basic definitions and concepts in Section 5.3.2. The lower bounds and the proof are given in Section 5.3.3.

\subsubsection{Definitions and concepts}

In this section, we give some definitions and concepts which are useful in deriving lower bounds for $\diamond$ values. We first classify each region $S_{j}$ in $S_{E}$ as either open or closed with respect to OPT. Then, extending the definitions of fragments and conjugates, we introduce conjugate fragments and delimiter fragments. Finally, we present a property of delimiter fragments in open regions.

Open and closed regions: With respect to OPT, a region $S_{j}$ in $S_{E}$ is said to be an open region if some $U U, A A$, or $U A$-substrings in $S_{j}$ are paired with some 2-substrings outside $S_{j}$; otherwise, it is a closed region.

Lemma 5.4 If $S_{m+1}$ is a closed region, then \#OPT $<h$. 
Proof. $\quad S_{m+1}$ has $3 n d$ more $A A$-substrings than $U U$-substrings. If $S_{m+1}$ is a closed region, these $3 n d A A$-substrings are not paired by OPT. Thus, $\diamond A A \geq 3 n d$. By Fact 5.3 , $\# \mathrm{OPT}<h+(n+1)+(2 m+2)-3 n d<h$.

Recall that $S_{E}$ is a sequence composed of $\delta$ 's, $\bar{\delta}$ 's, $\pi$ 's, and $\bar{\pi}^{\prime}$ 's. Each $\delta(k)$ (respectively $\overline{\delta(k)})$ consists of two substrings of the form $U^{+} A^{+}$, each of these substrings is called a fragment. Furthermore, each $\pi(k)$ (resp. $\overline{\pi(k)}$ ) consists of two substrings of the form $C^{+}$ (respectively $G^{+}$), each of these subtrings is also called a fragment.

Conjugate fragments and delimiter fragments: Consider any fragment $F$ in $S_{E}$. Another fragment $F^{\prime}$ in $S_{E}$ is called a conjugate fragment of $F$ if $F^{\prime}$ is the conjugate of $F$. Note that if $F$ is a fragment of a certian $\delta(k)$ (resp. $\pi(k)$ ), then $F^{\prime}$ appears only in some $\overline{\delta(k)}$ (respectively $\overline{\pi(k)}$ ), and vice versa. By construction, if $F$ is a fragment of some delimiter sequence $V_{j}$ or $W_{j}$, then $F$ has a unique conjugate fragment in $S_{E}$, which is located in $\overline{V_{j}}$ or $\overline{W_{j}}$, respectively. However, if $F$ is a fragment of some non-delimiter sequence, says, $\left\langle x_{i}\right\rangle$, then for every instance of $\left\langle\overline{x_{i}}\right\rangle$ in $S_{E}, F$ contains one conjugate fragment in $\left\langle\overline{x_{i}}\right\rangle$.

A fragment $F$ is said to be paired with its conjugate fragment $F^{\prime}$ by OPT if OPT includes all the pairs of bases between $F$ and $F^{\prime}$.

For $1 \leq j \leq m+1$, the fragment $F$ in $V_{j}$ or $W_{j}$ is called a delimiter fragment. Note that the delimiter fragment $F$ should be of the form $C^{2 d+k}$ for $2 d>k>0$.

The following lemma shows a property of delimiter fragments in open regions.

Lemma 5.5 If $S_{j}$ is an open region, then both delimiter fragments of either $V_{j}$ or $W_{j}$ must not pair with their conjugate fragments in OPT.

Proof. We prove the statement by contradiction. Suppose one fragment of $V_{j}$ and one fragment of $W_{j}$ are paired with their conjugate fragments. Let $\left(s_{x}, s_{x+1} ; s_{y-1}, s_{y}\right)$ and $\left(s_{x^{\prime}}, s_{x^{\prime}+1} ; s_{y^{\prime}-1}, s_{y^{\prime}}\right)$ be some particular stacking pairs in $V_{j}$ and $W_{j}$, respectively. Since $S_{j}$ is an open region, we can identify a stacking pair $\left(s_{x^{\prime \prime}}, s_{x^{\prime \prime}+1} ; s_{y^{\prime \prime}-1}, s_{y^{\prime \prime}}\right)$ where $s_{x^{\prime \prime}} s_{x^{\prime \prime}+1}$ and $s_{y^{\prime \prime}-1} s_{y^{\prime \prime}}$ are 2-substrings within and outside $S_{j}$, respectively. Note that these three stacking pairs form an interleaving block. By Lemma 2.1, OPT is not planar, reaching a contradiction.

\subsubsection{Proof of the only-if part}

By Lemma 5.4, it suffices to assume that $S_{m+1}$ is an open region. Before we give the proof of the only-if part, let us consider the following lemma.

Lemma 5.6 Let $\alpha$ be the number of delimiter fragments that are not paired with their conjugate fragments. Then, $\diamond C C+\diamond G G \geq \alpha+(\# G C-\diamond G C)$.

Proof. By construction, a $G C$-substring must be next to the left end of a delimiter fragment $F$, which is of the form $C^{+}$. No other $G C$-substrings can exist. If this $G C$-substring is paired, the leftmost $C C$-substring of $F$ must not be paired as there is no $G G C$ pattern in $S_{E}$. Thus, $F$ must be one of the $\alpha$ delimiter fragments that are not paired with their conjugate fragments. Based on this observation, we classify the $\alpha$ delimiter fragments into 


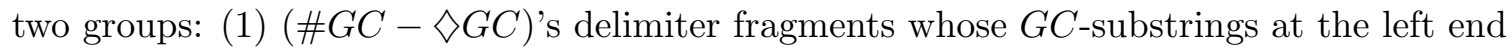
are paired; and $(2) \alpha-(\# G C-\diamond G C)$ 's delimiter fragments whose $G C$-substrings at the left end are not paired.

For each delimiter fragment $F=C^{2 d+k}$ in group (1), since the $G C$-substring on the left of $F$ is paired, the leftmost $C C$-substring of $F$ must not be paired by OPT. For the remaining $2 d+k-2 C C$-substrings, we either find a $C C$-substring which is not paired by OPT; or these $2 d+k-2 C C$-substrings are paired to $G G$-substrings in some fragment $F^{\prime}=G^{2 d+k^{\prime}}$ with $2 d>k^{\prime}>k$, and thus, some $G G$-substring of $F^{\prime}$ is not paired. Therefore, each delimiter fragment in group (1) introduces either (i) two unpaired $C C$-substrings or (ii) one unpaired $C C$-substring and one unpaired $G G$-substring. Hence, the total number of unpaired $C C$ and $G G$-substrings due to delimiter fragments in group (1) $\geq 2(\# G C-\diamond G C)$.

For each delimiter fragment $F=C^{2 d+k}$ in group (2), consider the $C C$-substrings in $F$. With a similar argument, we can show that each delimiter fragment in group (2) introduces either (i) one unpaired $C C$-substring or (ii) one unpaired $G G$-substring. Hence, the total number of unpaired $C C$ and $G G$-substrings due to delimiter fragments in group $(2) \geq \alpha-(\# G C-\diamond G C)$.

In total, we have $\diamond C C+\diamond G G \geq \alpha+(\# G C-\diamond G C)$.

Now, we state a lemma which shows the lower bounds for some $\diamond$ values in terms of the number of open regions in OPT.

Lemma 5.7 Let $\ell \geq 1$ be the number of open regions in OPT.

(1) If $S_{m+1}$ is an open region, then $\diamond U U \geq 3(m+1-\ell) d$.

(2) $\max \{\diamond C C, \diamond G G\} \geq \ell+(\# G C-\diamond G C) / 2$.

(3) If $\ell=n+1, S_{m+1}$ is an open region, and $E$ does not have a perfect matching, then either (a) $\diamond U U \geq 3(m-n) d+1$, (b) $\diamond A A \geq 1$, or (c) $\diamond U A \geq 2$.

Proof.

Statement 1. Within each closed region $S_{j}$ where $j \neq m+1,3 d$ 's $U U$-substrings cannot paired in OPT. As there are $m+1-\ell$ such closed regions, $3(m+1-\ell) d U U$-substrings are not paired in OPT. Thus, $\diamond U U \geq 3(m+1-\ell) d$.

Statement 2. By Lemma 5.5, we can identify $2 \ell$ fragments in $V_{j}$ and $W_{j}$ of all open regions which are not paired with their conjugate fragments. Then, by Lemma 5.6, we have $\diamond C C+\diamond G G \geq 2 \ell+(\# G C-\diamond G C)$. Thus, $\max \{\diamond C C, \diamond G G\} \geq \ell+(\# G C-\diamond G C) / 2$.

Statement 3. By a similar argument to the proof for Statement 1, within the $m+1-\ell=$ $m-n$ closed regions, $3(m-n) d U U$-substrings are not paired in OPT.

For the $\ell=n+1$ open regions, one of them must be $S_{m+1}$. Let $S_{j_{1}}, \ldots, S_{j_{n}}$ be the remaining $n$ open regions. Recall that $e_{j_{1}}, \ldots, e_{j_{n}}$ are the corresponding edges of these $n$ open regions. Since these $n$ edges cannot form a perfect matching, some node, says $x_{k}$, is adjacent to these $n$ edges more than once. Thus, within $S_{j_{1}}, \ldots, S_{j_{n}}, S_{m+1}$, we have more $\left\langle x_{k}\right\rangle$ than $\left\langle\overline{x_{k}}\right\rangle$. Therefore, at least two of the fragments in all $\left\langle x_{k}\right\rangle$ are not paired with their conjugate fragments. 
Let $F$ be one of such fragments. Note that $F$ is of the form $U^{d} A^{k}$. Since $F$ is not paired with its conjugate fragment, one of the following three cases occurs in OPT:

Case 1: An $U U$-substring of $F$ is not paired.

Case 2: An $A A$-substring of $F$ is not paired.

Case 3: All $U U$-substrings and $A A$-substrings $F$ are paired. In this case, $U^{d}$ of $F$ is paired with $A^{d}$ of a fragment $F^{\prime}=U^{k^{\prime}} A^{d}$; and $A^{k}$ of $F$ is paired with some substring $U^{k}$ of some fragment $F^{\prime \prime}$. As $F^{\prime}$ and $F^{\prime \prime}$ are not the same fragment, the $U A$-substrings of both $F$ and $F^{\prime}$ are not paired.

In summary, we have either $(1) \diamond U U \geq 3(m-n) d+1$, or $(2) \diamond A A \geq 1$, or $(3) \diamond U A \geq 2$.

Based on Lemma 5.7, we prove the only-if part by a case analysis in the following lemma.

Lemma 5.8 If $E$ does not have a prefect matching, then \#OPT $<h$.

Proof. Recall that if $S_{m+1}$ is a closed region, then \#OPT $<h$. Now, suppose that $S_{m+1}$ is an open region. We show \#OPT $<h$ in three cases $\ell<n+1, \ell>n+1$ and $\ell=n+1$.

Case 1: $\ell<n+1$. By Lemma 5.7 (1), $\diamond U U \geq 3(m+1-\ell) d$. By Fact 5.3, we can conclude that $\# \mathrm{OPT}=h+n+1+(2 m+2)-3(n+1-\ell) d \leq h+n+1+(2 m+2)-3 d<h$.

Case 2: $\ell>n+1$. By Lemma 5.7 (2), $\max \{\diamond C C, \diamond G G\} \geq \ell+(\# G C-\diamond G C) / 2$. By Fact 5.3, \#OPT $\leq h+n+1-\ell$, which is smaller than $h$ because $\ell>n+1$.

Case 3: $\ell=n+1$. By Lemma 5.7 (3), either (a) $\diamond U U \geq 3(m-n) d+1$, or (b) $\diamond A A \geq 1$, or $(\mathrm{c}) \diamond U A \geq 2$. By Fact 5.3, \#OPT $\leq h+n-\max \{\diamond C C, \diamond G G\}+(\# G C-\diamond G C) / 2$. By Lemma $5.7(2)$, we have \#OPT $<h$.

We conclude that if $E$ does not have a prefect matching, then \#OPT $<h$. Equivalently, if $\# \mathrm{OPT} \geq h$, then $E$ has a prefect matching.

\section{Conclusions}

In this paper, we have studied the problem of predicting RNA secondary structures that allow arbitrary pseudoknots with a simple free energy function that is minimized when the number of stacking pairs is maximized. We have proved that this problem is NP-hard if the secondary structure is required to be planar. We conjecture that the problem is also NP-hard for the general case. We have also given two approximation algorithms for this problem with worst-case approximation ratios of $1 / 2$ and $1 / 3$ for planar and general secondary structures, respectively. It would be of interest to improve these approximation ratios.

Another direction is to study the problem using energy function that is minimized when the number of base pairs is maximized. It is known that this problem can be solved in cubic time if the secondary structure can be non-planar [0]. However, the computational complexity of the problem is still open if the secondary structure is required to be planar. We conjecture that the problem becomes NP-hard under this additional condition. We 
would like to point out that the observation that have enabled us to visualize the planarity of stacking pairs on a rectangular grid does not hold in case of maximizing base pairs.

\section{References}

[1] T. Akutsu. Dynamic programming algorithms for RNA secondary structure prediction with pseudoknots. Discrete Applied Mathematics, 104(1-3):45-62, 2000.

[2] M. Garey and D. Johnson. Computers and Intractability: A Guide to the Theory of NP-Completeness. Freeman, New York, NY, 1979.

[3] R. Lyngs $\varnothing$, M. Zuker, and C. Pedersen. Internal loops in RNA secondary structure prediction. In Proceedings of the 3rd Annual International Conference on Computational Molecular Biology, pages 260-267, Lyon, France, 1999.

[4] R. B. Lyngs $\varnothing$ and C. N. S. Pedersen. RNA pseudoknot prediction in energy based models. Journal of Computational Biology, 7(3/4):409-428, 2000.

[5] R. B. Lyngs $\varnothing$, M. Zuker, and C. N. S. Pedersen. Fast evaluation of internal loops in RNA secondary structure prediction. Bioinformatics, 15(6):440-445, 1999.

[6] J. Meidanis and J. Setubal. Introduction to Computational Molecular Biology. International Thomson Publishing, New York, 1997.

[7] R. Nussinov, G. Pieczenik, J.R. Griggs, and D.J. Kleitman. Algorithms for loop matchings. SIAM Journal on Applied Mathematics, 35(1):68-82, 1978.

[8] E. Rivas and S. R. Eddy. A dynamic programming algorithm for RNA structure prediction including pseudoknots. Journal of Molecular Biology, 285(5):2053-2068, 1999 .

[9] M. Tompa. Lecture notes on biological sequence analysis. Technical Report \#200006-01, Department of Computer Science and Engineering, University of Washington, Seattle, 2000.

[10] Y. Uemura, A. Hasegawa, S. Kobayashi, and T. Yokomori. Tree adjoining grammars for RNA structure prediction. Theoretical Computer Science, 210(2):277-303, 1999.

[11] M. S. Waterman. Introduction to Computational Biology: Maps, Sequences and Genomes. Chapman \& Hall, New York, NY, 1995.

[12] M. Zuker. The use of dynamic algorithms in RNA secondary structure prediction. In M. S. Waterman, editor, Mathematical Methods for DNA Sequences, pages 159-184. CRC Press Inc., Boca Raton, FL, 1989.

[13] M. Zuker and D. Sankoff. RNA secondary structures and their prediction. Bulletin of Mathematical Biology, 46(4):591-621, 1984. 